\title{
INTERACTION OF GAS FRONTS
}

\author{
BY \\ A. A. LACEY (Heriot-Watt University, Edinburgh, United Kingdom) \\ AND

\section{J. L. VAZQUEZ (Universidad Autónoma de Madrid, Spain)}

\begin{abstract}
During rock blasting separate charges initiate movement of gas at high pressure through fractured rock. This gas occupies regions of compact support enclosed by moving boundaries, the gas fronts, at which the pressure is effectively zero. Here we consider the local behaviour of such gas fronts, albeit taking the system to be rather simpler so that the gas flow can be modelled by the porous medium equation, when two identically pressurized regions interact and when a gas front impinges upon a fixed boundary where the pressure is zero.
\end{abstract}

1. Introduction. To quarry rock by cliff blasting the detonation of explosive charges act as point sources of high pressure gas. The gas then flows through fractured rock according to a highly nonlinear diffusion law in such a way as to occupy regions that are expanding but bounded at any instant. At the (free) boundaries of these, the gas fronts or blast fronts, the pressure is much smaller (e.g., atmospheric) and can be taken to be zero. For the full model of this and its derivation see [2]. As a rather simpler prototype we consider here the porous medium equation that applies to the flow of compressible gas moving relatively slowly so that Darcy's law applies:

$$
\frac{\partial u}{\partial t}=\nabla \cdot\left(u^{m} \nabla u\right)
$$

This has similar properties to the more complex rock blasting model. To simplify the work still further we shall only take the case of $m=1$ and restrict ourselves to two dimensions. We note that all the results will carry over with trivial modifications to general $m>0$ and to $N>1$ dimensions. We now have

$$
\frac{\partial u}{\partial t}=\nabla \cdot(u \nabla u)=u \Delta u+|\nabla u|^{2} .
$$

The solution to (2) (and indeed (1)) originating from a point source at a time $t_{0}$ is the Barenblatt-Pattle similarity solution (see, e.g., $[1,5]$; here

$$
u=\left(t-t_{0}\right)^{-1 / 2}\left(C-\frac{1}{8}\left(t-t_{0}\right)^{-1 / 2} r^{2}\right)_{+}
$$

Received October 10, 1990.

The second author was supported by SERC grant GR/D/73096. 
for $t>t_{0}$, where $C$ is a measure of the strength of the initial source (blast) and $r$ is the distance from that point. We see that $u>0$ for $r<R(t)=\left[8 C\left(t-t_{0}\right)^{1 / 2}\right]^{1 / 2}$ and $u=0$ for $r \geq R(t)$.

We wish to know, first, what happens when a blast, such as that represented by (3), impinges upon a cliff place. This will be represented by a fixed flat boundary on which $u=0$ (pressure is maintained at atmospheric). For convenience we take this boundary to be at $x=0$,

$$
u=0 \quad \text { on } x=0 .
$$

We also take the source to be at $x=D>0, y=0, t=t_{0}<0$, and $C=$ $\frac{1}{8}\left(-t_{0}\right)^{-1 / 2} D^{2}$ so that the solution (3) applies for $t_{0}<t \leq 0$ and the moving boundary meets the fixed boundary at $x=y=t=0$. Denoting the free boundary for $t \geq 0$ by $\Gamma(t)$,

$$
\Gamma(t)=\partial \Omega(t) \cap\{(x, y): x>0\},
$$

where $\Omega(t)=\operatorname{supp} u(\cdot, \cdot, t)$, we shall estimate $l(t)$, where $(x, y)=(0, \pm l(t))$ are the end points of $\Gamma$, i.e., $\partial \Omega=\Gamma \cup\{(0, y):-l \leq y \leq l\}$. Certainly $l(0)=0$ and we expect $l(t)>0$ for $t>0$ (see Figs. 1 and 2).

Second, we wish to estimate the local behaviour when two identical blast waves meet. Taking these to originate at $x= \pm D, y=0, t=t_{0}<0$, and again, $C=\frac{1}{8}\left(-t_{0}\right)^{-1 / 2} D^{2}$, the problem is symmetric about $x=0$ so that it is again possible to consider (2) only in $x>0$ with

$$
\frac{\partial u}{\partial n}=\frac{\partial u}{\partial x}=0 \quad \text { on } x=0 .
$$

We may define $l(t)$ for $t>0$ as in the above Dirichlet problem but for $t>0$ we now expect $\Omega$ and $\Gamma$ to be more like those illustrated in Fig. 3.

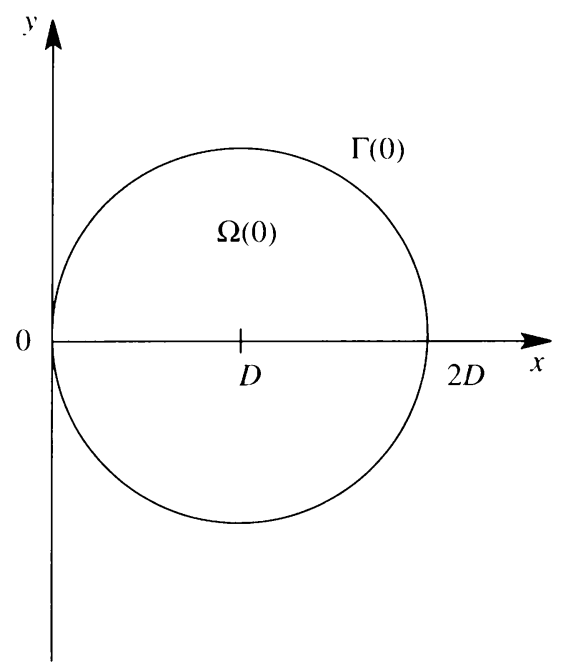

FIG. 1. Support of $u$ at $t=0$. 


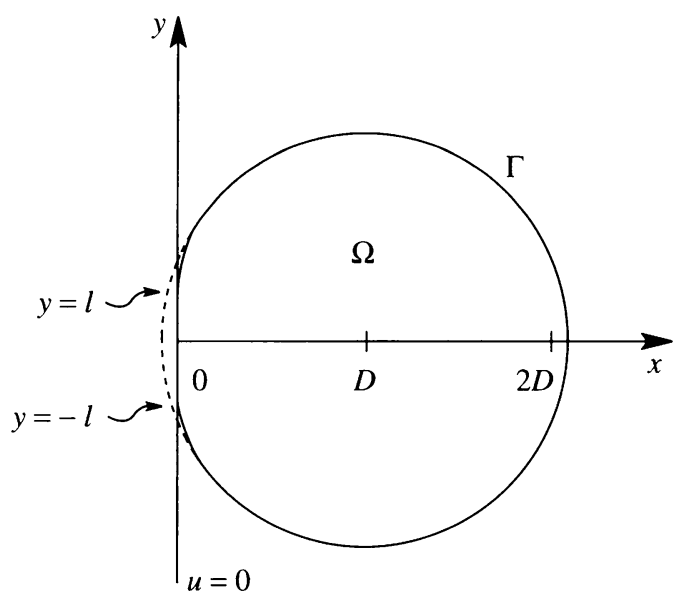

Fig. 2. Support of $u$ for the Dirichlet problem when $t>0$.

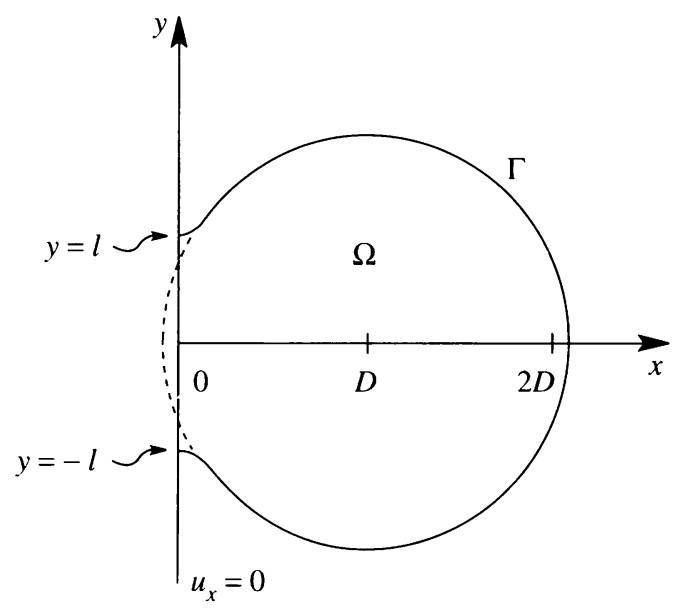

Fig. 3. Support of $u$ for the Neumann problem when $t>0$.

The normal velocity of the free boundary is known to be given by

$$
v=-\frac{\partial u}{\partial n}
$$

so that for $t_{0}<t<0$ the boundary speed is

$$
v=\dot{R}=(C / 2)^{1 / 2}\left(t-t_{0}\right)^{-3 / 4},
$$

giving a local speed at $t=0-$ of

$$
V=(C / 2)^{1 / 2}\left(-t_{0}\right)^{-3 / 4}=D /\left(-4 t_{0}\right) .
$$

Near the point of contact, 0 , between $\Gamma(0)$ and the fixed boundary $x=0$, the free boundary $\Gamma(0)$ is given by $x=d(y)$ where $d(y)$ is defined by $D-\left(D^{2}-y^{2}\right)^{1 / 2}$. We thus have $d(y) \sim y^{2} /(2 D)$ for $y$ small. 
Formal asymptotic analysis then suggests that for $y$ small the time taken for $\Gamma$ to reach $(0, y)$ is given by $t \sim d(y) / V \sim 2 y^{2}\left(-t_{0}\right) / D^{2}$, i.e., $l(t) \sim d^{-1}(V t) \sim \sqrt{2 D V t}$ for $t$ small, for both the Dirichlet and Neumann problems. It is the aim of the present paper to prove that these estimates are indeed correct for (2) with either boundary condition (4) or (5), in each case subject to the initial condition

$$
u(x, y, 0)=\frac{1}{8}\left(-t_{0}\right)^{-1}\left(2 D x-x^{2}-y^{2}\right)=\frac{V}{2 D}\left(2 D x-x^{2}-y^{2}\right)
$$

(recalling that $r^{2}=(x-D)^{2}+y^{2}, C=\frac{1}{8}\left(-t_{0}\right)^{-1 / 2} D^{2}, V=D /\left(-4 t_{0}\right)$ ).

2. Main results. We may define

$$
U=\left(t-t_{0}\right)^{-1 / 2}\left[C-\frac{1}{8}\left(t-t_{0}\right)^{-1 / 2} r^{2}\right]_{+}, \quad t>t_{0}
$$

with $t_{0}, C$, and $r$ as above.

Then $U$ satisfies (2), $U=u$ for $t \leq 0$ and $U \geq 0$ on $x=0$. Thus $U$ is an upper solution for (2), (4), (6) and we immediately get that $l(t) \leq \sup \{y: U(0, y, t)>0\}$. From Definition (7) we know that $U>0$ for $y^{2}+x^{2}-2 D x<8 C\left(t-t_{0}\right)^{1 / 2}-D^{2}$ and so for $0<t \ll 1, U>0$ if $x=0$ and $y^{2} \leq 4 C\left(-t_{0}\right)^{1 / 2} t+O\left(t^{2}\right)=2 D V t+O\left(t^{2}\right)$. Consequently we have

LemMa 1. For $0<t \ll 1, l(t) \lesssim \sqrt{2 D V t}$, i.e., $d(l(t)) \lesssim V t$, for problem (2), (4), (6) .

We next obtain a complementary estimate:

LEMMA 2. For the problem (2), (4), (6), if $0<t \ll 1$ then $l(t) \gtrsim \sqrt{2 D V t}$, i.e., $d(l(t)) \geqslant V t$.

Proof. We compare $u$ with another self-similar (Barenblatt-Pattle) solution to (2) with centre $(X, Y), w$, such that at $t=0$ the support of $w$ lies within that of $u$, the boundaries of the two supports are tangent at some point, at which they have the same gradient $V$, so that $w \leq u$ (see Fig. 4) at $t=0$. By the maximum principle the same inequality holds for $t>0$.

Now $w=\left(t-t_{1}\right)^{-1 / 2}\left[B-\frac{1}{8}\left(t-t_{1}\right)^{-1 / 2} r_{1}^{2}\right]_{+}$where $r_{1}$ denotes distance from $(X, Y),-t_{1}=E /(4 V), E$ is the radius of the support at $t=0$, i.e., $E=$ $D-\sqrt{(D-X)^{2}+Y^{2}}$ and $B=\frac{1}{8}\left(-t_{1}\right)^{-1 / 2} E^{2}$. The support of $w$ reaches $x=0$ at $y=Y$ when

$$
t=t_{1}+\left(X^{2} / 8 B\right)^{2}=t_{1}\left(1-X^{4} / E^{4}\right) \simeq-2 t_{1} Y^{2} /(X(D-X)) \text { for } Y \ll 1
$$

since for $Y \ll 1$ and $0<X<D$ we have $E \simeq X-Y^{2} /(2(D-X))$. We conclude that $t \simeq-2 t_{1} Y^{2} /(X(D-X)) \simeq Y^{2} /(2 V(D-X))$ if $Y^{2} \ll X(D-X)$.

Given any $X<D$ we see that $u(0, y, t)>0$ for $t \gtrsim y^{2} /(2 V(D-X))$ if $y \ll 1$. It follows that $l(t) \gtrsim \sqrt{2(D-X) V t}$ for $t \ll 1$.

Since this holds for arbitrary $X$, we may let $X \rightarrow 0$ to obtain $l(t) \geq \sqrt{2 D V t}+$ $o\left(t^{1 / 2}\right)$ for $0<t \ll 1$. 


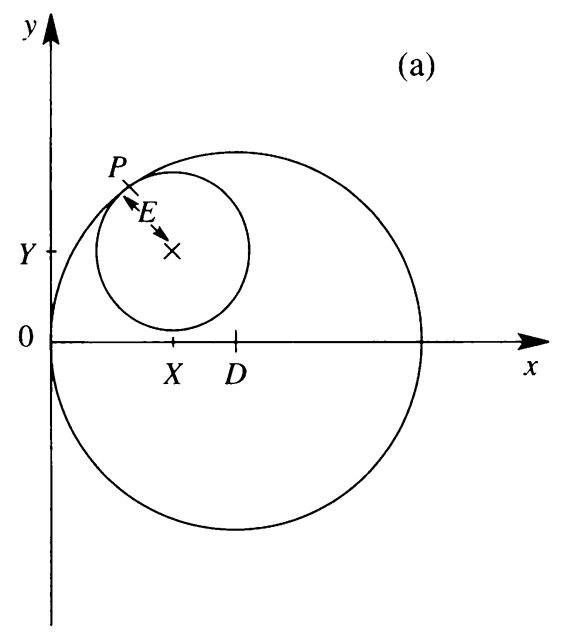

Fig. 4. (a) Supports of $u$ and the comparison function $w$ at $t=0$.

(b) Section through $P,(X, Y),(D, 0)$ showing $u$ and $w$.

From these lemmas we have immediately

THEOREM 1. Let $u$ be the solution of the porous medium equation (2) subject to the initial condition $u=\frac{V}{2 D}\left(2 D x-x^{2}-y^{2}\right)_{+}$at $t=0$ in $x>0$ and the Dirichlet boundary condition $u=0$ on $x=0$. Let $\Omega(t)$ be the support of $u$ at time $t \geq 0$ and $l(t)$ the distance covered by the support along the edge $x=0$, i.e., $\partial \Omega(t) \cap\{(0, y)\}=\{(0, y):-l \leq y \leq l\}$. Then $l(t)$ satisfies $l(t) \sim \sqrt{2 D V t}$ for $0<t \ll 1$.

This means that the time taken for the boundary of $\Omega(t)$ to reach $(0, y), t=$ $\omega(y)$, is given by $\omega(y) \sim d(y) / V$ for $y \ll 1$. Here $x=d(y) \sim y^{2} /(2 D)$ describes $\partial \Omega(0)$ locally near 0 and $V$ is the local boundary speed (i.e., boundary speed for $t \rightarrow 0-)$.

We turn now to the Neumann problem (2), (5), (6). We can again use $U$, given by (7), as a comparison function. Noting that $\partial U / \partial x>0$ on $x=0$ where $U>0$, we see that $U$ is now a strict lower solution so $u>0$ in the support of $U$ for $t>0$. Thus

Lemma 3. For $0<t \ll 1, l(t) \approx \sqrt{2 D V t}$, i.e., $d(l(t)) \approx V t$, for the problem (2), (5), (6).

The corresponding estimate from above turns out to be the most technical.

Lemma 4. For the problem (2), (5), (6) if $0<t \ll 1$ then $l(t) \lesssim \sqrt{2 D V t}$, i.e., $d(l(t)) \lesssim V t$.

Proof. Writing $X(x)=D x-x^{2} / 2$, the initial condition (6) becomes

$$
u(x, y, 0)=\frac{V}{D}\left[X(x)-\frac{y^{2}}{2}\right]_{+} .
$$


We consider the function $z$,

$$
z(x, y, t)=\frac{V}{D}\left[\left(X^{2}+c t^{2}\right)^{1 / 2}+b t-\frac{y^{2}}{2}\right]_{+},
$$

for $0 \leq x \leq 2 D$, where $b$ and $c$ are positive constants to be chosen. It is readily seen that in the support of $z$

$$
\begin{aligned}
& \frac{\partial z}{\partial t}=\frac{V}{D}\left[b+c t\left(X^{2}+c t^{2}\right)^{-1 / 2}\right], \\
& \frac{\partial z}{\partial x}=\frac{V}{D}\left(x^{2}+c t^{2}\right)^{-1 / 2} X X^{\prime}=0 \text { for } x=0 \text { and for } x=2 D,
\end{aligned}
$$

and

$$
\begin{aligned}
& z \Delta z+|\nabla z|^{2}=\frac{V^{2}}{D^{2}}\left\{\left[\left(X^{2}+c t^{2}\right)^{1 / 2}+b t-\frac{y^{2}}{2}\right]\right. \\
& \times\left[\left(X^{2}+c t^{2}\right)^{-1 / 2}\left(X X^{\prime \prime}+X^{\prime 2}\right)-\left(X^{2}+c t\right)^{-3 / 2} X^{2} X^{\prime 2}-1\right] \\
& \left.+\left(X^{2}+c t^{2}\right)^{-1} X^{2} X^{\prime 2}+y^{2}\right\} \\
& <\frac{V^{2}}{D^{2}}\left\{\left[\left(X^{2}+c t^{2}\right)^{1 / 2}+b t-\frac{y^{2}}{2}\right]\right. \\
& \times\left[\left(X^{2}+c t^{2}\right)^{-1 / 2} X^{\prime 2}-\left(X^{2}+c t^{2}\right)^{-3 / 2} X^{2} X^{\prime 2}\right] \\
& \left.+\left(X^{2}+c t^{2}\right)^{-1} X^{2} X^{\prime 2}+y^{2}\right\} \\
& \text { since } z>0 \text { and } X X^{\prime \prime}<0 \text {, } \\
& \leq \frac{V^{2}}{D^{2}}\left\{\left[\left(X^{2}+c t^{2}\right)^{1 / 2}+b t\right]\left[\left(X^{2}+c t^{2}\right)^{-1 / 2} X^{\prime 2}-\left(X^{2}+c t^{2}\right)^{-3 / 2} X^{2} X^{\prime 2}\right]\right. \\
& \left.+\left(X^{2}+c t^{2}\right)^{-1} X^{2} X^{\prime 2}+y^{2}\right\} \\
& \text { since } X^{2} /\left(X^{2}+c t^{2}\right)<1 \\
& =\frac{V^{2}}{D^{2}}\left\{X^{\prime 2}+b t\left(X^{2}+c t^{2}\right)^{-1 / 2} X^{\prime 2}-b t\left(X^{2}+c t^{2}\right)^{-3 / 2} X^{2} X^{\prime 2}+y^{2}\right\} \\
& <\frac{V^{2}}{D^{2}}\left\{X^{\prime 2}+b t\left(X^{2}+c t^{2}\right)^{-1 / 2} X^{\prime 2}+y^{2}\right\} \text {. }
\end{aligned}
$$

Choosing $b>D V, c=D V b$, we see that

$$
\frac{\partial z}{\partial t}>z \Delta z+|\nabla z|^{2}
$$

since $\left|X^{\prime}\right| \leq D$, provided that $y^{2} \leq D b / V-D^{2}$.

For $y^{2}>D b / V-D^{2}$ and taking $t$ small we need only consider $x$ bounded away from 0 and $2 D$ as otherwise $z=0$. But then $\partial z / \partial t$ and $z \Delta z+|\nabla z|^{2}$ are 
continuous in this part of the support of $z$. Moreover $\partial z / \partial t=b V / D$ and

$$
z \Delta z+|\nabla z|^{2}=\frac{V^{2}}{D^{2}}\left[\left(X-\frac{y^{2}}{2}\right)\left(X^{\prime \prime}-1\right)+X^{\prime 2}+y^{2}\right] \leq V^{2} \quad \text { at } t=0 .
$$

Again we see that the choice of $b>D V$ ensures that $\partial z / \partial t>z \Delta z+|\nabla z|^{2}$ for sufficiently small $t$.

Noting that $z=u$ at $t=0$ we see that $z$ is an upper solution for $u$ so that $u \leq z$, at least for short enough time, say $t \leq \tau$. This gives $l(t)^{2} \leq 2\left[b+(b D V)^{1 / 2}\right] t$, so by taking $b$ arbitrarily close to $D V$, we obtain the preliminary bound $l(t)^{2} \lesssim 4 D V t$ for $0<t \ll 1$. We now obtain a stronger estimate from an improved upper solution.

Motivated by the form of similarity solutions to one-dimensional problems (see, e.g., [4]) we define $w$ by

$$
w(x, y, t)= \begin{cases}\frac{V}{D}\left(D x+a t-y^{2} / 2\right)_{+} & y^{2} \geq 2 a t, \\ \frac{V}{D}\left(a t-y^{2} / 2\right) F\left(D x /\left(a t-y^{2} / 2\right)\right) & y^{2}<2 a t,\end{cases}
$$

where $a$ is some constant, $a>b$. Here $F(\eta)$ satisfies

$$
\begin{gathered}
F F^{\prime \prime}+F^{\prime 2}+\eta F^{\prime}-F=0 \quad \eta>0, \\
F^{\prime}(0)=0, \\
F(\eta) \sim \eta \quad \text { as } \eta \rightarrow \infty .
\end{gathered}
$$

The significance of $F$ lies in the fact that if $v(x, t)=t F(x / t)$ then $v$ is the solution to

$$
\begin{array}{ll}
\frac{\partial v}{\partial t}=\frac{\partial}{\partial x}\left(v \frac{\partial v}{\partial x}\right) & x>0, t>0, \\
\frac{\partial v}{\partial x}=0 \quad \text { on } x=0, & v=x \quad \text { at } t=0 .
\end{array}
$$

Before proceeding to use $w$ we must first get certain properties of $F$. Replacing the condition at infinity (10) by $F(0)=1$ we would have a solution to (8), (9) with $F>0, F^{\prime}>0$, and, since

$$
F^{\prime \prime \prime} / F^{\prime \prime}+\left(3 F^{\prime}+\eta\right) / F=0,
$$

$F^{\prime \prime}>0$. Then $\left(F-\eta F^{\prime}\right)^{\prime}<0$ so $F-\eta F^{\prime}=F^{\prime 2}+F F^{\prime \prime}$ decreases from 1 at $\eta=0$ to some $k, 0 \leq k<1$ as $\eta \rightarrow \infty$. Now $F^{\prime} / \eta-F / \eta^{2} \sim-k / \eta^{2}$ and it follows that $F(\eta)=A \eta+k+o(1)$ and $F^{\prime}(\eta)=A+o(1)$ as $\eta \rightarrow \infty$, where $A>0$ since $F^{\prime \prime}>0$. Again from (8) $F^{\prime \prime}=\left(F-\eta F^{\prime}-F^{\prime 2}\right) / F \sim\left(k-A^{2}\right) /(A \eta)$ as $\eta \rightarrow \infty$, which shows that $k=A^{2}$ since $F^{\prime}$ has already been seen to be bounded.

We may now rescale $F$ with $A^{2}$ and $\eta$ with $A$ to obtain a solution to (8), (9), (10), that satisfies

$$
\begin{aligned}
F>0, \quad F^{\prime}>0, & F^{\prime \prime}>0, \quad F(0)>1, \quad 1<F-\eta F^{\prime}<F(0) ; \\
F(\eta) \sim \eta+1+o(1), & F^{\prime}(\eta) \sim 1+o(1), \quad F-\eta F^{\prime}(\eta) \rightarrow 1 \quad \text { as } \eta \rightarrow \infty .
\end{aligned}
$$

From (11) we see that $F^{\prime \prime \prime} / F^{\prime \prime} \rightarrow-1$ as $\eta \rightarrow \infty$ so $F^{\prime \prime}$ must be exponentially small for large $\eta$. Hence $\eta^{2} F F^{\prime \prime} \rightarrow 0$ as $\eta \rightarrow \infty$ and is bounded. 
We now have that $w, \partial w / \partial y$, and hence $\nabla w$, are continuous (as is $\partial w / \partial t$ ). For $y^{2}>2 a t$ and where $w>0$,

$$
\begin{aligned}
\frac{\partial w}{\partial t}-w \Delta w-|\nabla w|^{2} & =\frac{V}{D}\left[a+\frac{V}{D}\left(x+a t-\frac{y^{2}}{2}\right)-D V-\frac{V}{D} y^{2}\right] \\
& >0 \text { for } y^{2}<(a-D V) D / V .
\end{aligned}
$$

On the other hand, for $y^{2}<2 a t$,

$$
\begin{aligned}
\frac{\partial w}{\partial t}-w \Delta w-|\nabla w|^{2}= & \frac{V}{D} a\left(F-\eta F^{\prime}\right)-V^{2}\left(F F^{\prime \prime}+F^{\prime 2}\right) \\
& -\frac{V^{2}}{D^{2}}\left(a t-\frac{y^{2}}{2}\right)\left[\frac{y^{2} \eta^{2} F^{\prime \prime}}{a t-y^{2} / 2}+\eta F^{\prime}-F\right] F \\
& -\frac{V^{2}}{D^{2}} y^{2}\left(\eta F^{\prime}-F\right)^{2} \quad\left(\text { where } \eta=\frac{D x}{a t-y^{2} / 2}\right) \\
= & \frac{V}{D}(a-D V)\left(F-\eta F^{\prime}\right) \\
& +\frac{V^{2}}{D^{2}}\left\{\left(a t-\frac{y^{2}}{2}\right)\left(F-\eta F^{\prime}\right) F-y^{2}\left[\left(F-\eta F^{\prime}\right)^{2}+\eta^{2} F F^{\prime \prime}\right]\right\}
\end{aligned}
$$

$>0$

on choosing $y$ small enough, say $|y|<\beta<[(a-D V) D / V]^{1 / 2}$ (recalling that $a>$ $b>D V)$.

Choosing some $\alpha, 0<\alpha<2 D$, we also have

$$
\begin{aligned}
\text { at } t=0, \quad u=\frac{V}{D}\left(D x-\frac{x^{2}}{2}-\frac{y^{2}}{2}\right)_{+} \leq \frac{V}{D}\left(D x-\frac{y^{2}}{2}\right)=w ; \\
\text { on } x=0, \quad \frac{\partial u}{\partial x}=0=F^{\prime}(0)=\frac{\partial w}{\partial x} ; \\
\text { on } y= \pm \beta, \\
u \leq z=\frac{V}{D}\left[\left(X^{2}+D V b t\right)^{1 / 2}+b t-\frac{y^{2}}{2}\right]_{+} \leq \frac{V}{D}\left(D x+a t-\frac{y^{2}}{2}\right)_{+}=w
\end{aligned}
$$

on $x=\alpha, u \leq z \leq w$ as above for $y^{2} \geq 2 a t$ and where $y^{2}<2 a t$,

$$
u \leq z=\frac{V}{D}\left[\left(X^{2}+D V b t\right)^{1 / 2}+b t-\frac{y^{2}}{2}\right]_{+} \leq \frac{V}{D}\left(a t-\frac{y^{2}}{2}\right) F\left(\frac{D \alpha}{a t-y^{2} / 2}\right)=w
$$

again taking $t$ sufficiently small.

Hence for some $T, 0<T<\tau$, if $0 \leq t \leq T, 0 \leq x \leq \alpha,-\beta \leq y \leq \beta$, then $w$ is an upper solution for (2), (5), (6), i.e., $u \leq w$.

But $w=0$ for $y^{2} \geq 2 a t$ and we deduce that $l(t)^{2} \leq 2 a t$. 
Taking $a$ arbitrarily close to $D V$ it is seen that $l(t) \lesssim \sqrt{2 D V t}$ for $0<t \ll 1$. The following result comes immediately from Lemmas 3 and 4 .

THEOREM 2. Let $u$ be the solution of the porous medium equation (2) in $x>0$ subject to the initial condition $u=\frac{V}{2 D}\left(2 D x-x^{2}-y^{2}\right)_{+}$at $t=0$ and the Neumann boundary condition $\partial u / \partial x=0$ on $x=0$; then $l(t)$, as given previously, satisfies $l(t) \sim \sqrt{2 D V t}$ for $0<t \ll 1$. Equivalently, writing the boundary of the support of $u$ at $t=0$ in the form $x=d(y)$ locally near 0 , the time taken for the support to reach $(0, y), t=\omega(y)$, satisfies $\omega(y) \sim d(y) / V$ for $y$ small. Here $V$ is the local boundary speed $(|\nabla u|$ at $x=y=t=0)$.

3. Discussion. We may note that the result that the time $t=\omega(y)$ taken for the support (blast wave) to reach the point $y$ on the fixed boundary $x=0$ is given by $\omega \sim d / V$, where the free boundary is locally $x=d(y)$ near 0 and $V$ is the local boundary speed $\left(u_{x}\right.$ at 0 with $t=0$ for (2)), may be obtained in more general situations:

(i) For other porous medium equations $(m>0$ in (1)) and in $N>1$ dimensions. The above arguments carry through with the modification that the Barenblatt-Pattle solution originating from $r=0$ and $t=0$ becomes

$$
u=t^{-N /(N m+2)}\left[C-\frac{m}{2(N m+2)} r^{2} t^{-2 /(N m+2)}\right]_{+}^{1 / m} .
$$

Here the radius of support is $R(t)=\left[(2 \mathrm{C} / \mathrm{m})(\mathrm{Nm}+2) t^{2 /(N m+2)}\right]^{1 / 2}$ and the boundary moves with speed

$$
\dot{R}=-\frac{1}{m} \frac{\partial}{\partial n}\left(u^{m}\right)=\left[\frac{2 C}{m(N m+2)}\right]^{1 / 2} t^{-(N m+1) /(N m+2)} .
$$

In particular, for the solution originating from $x_{1}=D>x_{2}=\cdots=x_{N}=0$ at $t=t_{0}<0$, which reaches $x=0$ when $t=0$,

$$
\dot{R}=V=\left[\frac{2 C}{m(N m+2)}\right]^{1 / 2}\left(-t_{0}\right)^{-(N m+1) /(N m+2)}=\frac{D}{(N m+2)\left(-t_{0}\right)}
$$

at that time and

$$
C=\left[\frac{D^{2} m}{2(N m+2)}\right]\left(-t_{0}\right)^{-2 /(N m+2)} .
$$

The initial condition at $t=0$ is thus $u=\left[V\left(D^{2}-r^{2}\right) /(2 D)\right]_{+}^{1 / m}$, where $r=$ distance from $(D, 0, \ldots, 0)$.

Lemmas 1, 2, and 3 and Theorems 1 and 2 carry over precisely as before. The proof of Lemma 4 only requires the change of comparison functions,

$$
\left.z=\left\{\frac{V}{D}\left(X^{2}+c t^{2}\right)+b t-y^{2} / 2\right]\right\}_{+}^{1 / m}
$$

and

$$
w= \begin{cases}\left\{\frac{V}{D}\left(D x_{1}+a t-y^{2} / 2\right)\right\}_{+}^{1 / m} & \text { for } y^{2} \geq 2 a t, \\ \left\{\frac{V}{D}\left(a t-y^{2} / 2\right) F\left(D x /\left(a t-y^{2} / 2\right)\right)\right\}^{1 / m} & \text { for } y^{2}<2 a t,\end{cases}
$$


with $X=D x_{1}-x_{1}^{2} / 2, y^{2}=x_{2}^{2}+\cdots+x_{N}^{2}$ and $F(\eta)$ satisfying $F F^{\prime \prime}+F^{\prime 2} / m+$ $\eta F^{\prime}-F=0, \eta>0 ; F^{\prime}(0)=0 ; F(\eta) \sim \eta$ as $\eta \rightarrow \infty$.

(ii) For a Robin boundary condition, $\partial u / \partial x=\gamma u$ on $x=0,0 \leq \gamma \leq \infty$, since we can bound the solution to this problem from each side by the solutions to the Dirichlet and Neumann problems.

(iii) For rather more general initial data the lemmas should follow in a similar fashion as long as the initial data and the initial free boundary, $\Gamma(0)$, are reasonably smooth (say $C^{2}$ for $\left.\Gamma(0)\right)$ near 0 .

We conjecture that the above estimates apply for cases of interaction with fixed boundaries that although not necessarily flat are still smooth. Similar results should also hold for more general degenerate nonlinear diffusion such as those with diffusivity depending upon the gradient of $u$, as in the actual model for rock blasting, $u_{t}=\nabla \cdot\left(f(x) u^{m}|\nabla u|^{\gamma-1}\right)$. For the homogeneous problem (see [3]), $f(x) \equiv 1$, selfsimilar solutions $u=t^{\alpha}\left[C-B r^{b} / t^{\beta}\right]_{+}^{a}$, where $a=\gamma /(m+\gamma-1), b=1+1 / \gamma, B=$ $(m+\gamma-1) K^{-1 / \gamma} /(\gamma+1), \alpha=-N / K, \beta=(\gamma+1) / K$, and $K=N(m+\gamma-1)+\gamma(\gamma+1)$, can be used in the same way as the Barenblatt-Pattle solutions in Lemmas 1, 2, and 3.

Finally, it must be noted that our present results, even with the possible generalizations just mentioned, almost always fail to cover cases of asymmetric interaction of gas fronts.

Acknowledgment. The authors wish to thank the SERC for financial support.

\section{REFERENCES}

[1] G. I. Barenblatt, On the self-similar solutions of the Cauchy problem for nonlinear parabolic equation of nonstationary filtration, Prikl. Math. Mech. 20, 761-763 (1956)

[2] H. E. Erhie, D. Phil. thesis, Oxford University, 1988

[3] J. R. Esteban and J. L. Vazquez, Homogeneous diffusion in $\mathbb{R}$ with power like nonlinear diffusivity, Arch. Rat. Mech. Anal. 103, 39-80 (1988)

[4] R. E. Grundy, A mathematical model for rock blasting involving a degenerate nonlinear diffusion equation, Quart J. Mech. Appl. Math. 43, 173-188 (1990)

[5] R. E. Pattle, Diffusion from an instantaneous point source with concentration dependent coefficient, Quart J. Mech. Appl. Math. 12, 407-409 (1959) 